\title{
Measurements of scalar power spectra in high Schmidt number turbulent jets
}

\author{
By PAUL L. MILleR $\dagger$ and PAUL E. DIMOTAKis \\ Graduate Aeronautical Laboratories, California Institute of Technology, \\ Pasadena, CA 91125, USA
}

(Received 4 March 1995 and in revised form 2 September 1995)

\begin{abstract}
We report on an experimental investigation of temporal, scalar power spectra of round, high Schmidt number $\left(S c \simeq 1.9 \times 10^{3}\right)$, momentum-dominated turbulent jets, for jet Reynolds numbers in the range of $1.25 \times 10^{4} \leqslant R e \leqslant 7.2 \times 10^{4}$. At intermediate scales, we find a spectrum with a slope (logarithmic derivative) that increases in absolute value with Reynolds number, but remains less than $5 / 3$ at the highest Reynolds number in our experiments. At the smallest scales, our spectra exhibit no $k^{-1}$ power-law behaviour, but, rather, seem to be approximated by a log-normal function, over a range of scales exceeding a factor of 40 , in some cases.
\end{abstract}

\section{Introduction}

We report on an experimental investigation of temporal scalar power spectra of round, high Schmidt number, turbulent jets. In these experiments, the jet-fluid concentration (scalar) power spectra were examined for several reasons. Spectra are sensitive diagnostics of the flow, providing information over a wide range of scales. Historically, they have been the object of a great deal of attention, partially because it is possible to extract predictions for spectral slopes from various turbulence theories and models.

Key among such turbulence theories are the 1941 paper by Kolmogorov (1941), with implications for the scalar field in the inertial range discussed by Corrsin (1951), Oboukhov (1962), and, for higher wavenumbers, the theory by Batchelor (1959). See, for example, discussions in Monin \& Yaglom (1975), as well as in the recent review by Gibson (1991). Both the Corrsin and the Oboukhov theories yield predictions of power-law spectra and of the spectral power-law logarithmic derivative, or slope, as it will be subsequently referred to in this paper. Specifically, the Corrsin and Oboukhov theories predict a scalar spectrum proportional to $k^{-5 / 3}$ in the inertial range, as did the 1941 Kolmogorov theory for the energy spectrum.

For energy spectra, experimental confirmation of these predictions has been documented in the past (cf. compilation of data by Chapman 1979). Recent experiments by Saddoughi \& Veeravalli (1994), in high Reynolds number turbulent boundary layers, however, indicate that a $-5 / 3$ power-law regime is only approached at the highest Reynolds numbers. Specifically, longitudinal velocity spectra recorded at the mid-layer $\left(y^{+} \approx 1.6 \times 10^{4}\right)$ of a turbulent boundary exhibit less than a decade of $-5 / 3$ range at a local Taylor Reynolds number of $R e_{T} \simeq 600$ (outer flow Reynolds number

† Current address: Lawrence Livermore National Laboratory; PO Box 808, L-022; Livermore, CA 94551, USA. 
of $\left.\operatorname{Re}_{\delta} \equiv U_{e} \delta_{99} / v \simeq 7.3 \times 10^{5}\right)$, with about a decade of $-5 / 3$ range at $R e_{T} \simeq 1450$ $\left(R e_{\delta} \simeq 3.6 \times 10^{6}\right)$. Saddoughi \& Veeravalli (1994) may also be consulted for citations of other recent measurements and theoretical discussions on turbulent energy spectra.

The situation is less clear concerning scalar spectra, with departures from the predicted behaviour continuing to fuel debate about details and refinements of the theory. Batchelor (1959) and Batchelor, Howells \& Townsend (1959) recognized that for Schmidt, or Prandtl, numbers away from unity there exists an additional, scalar-diffusion, scale, now referred to as the Batchelor scale, which admits a change in the scalar spectral behaviour. The Batchelor theory predicted that the scalar power spectrum at high Schmidt numbers would display a $k^{-1}$ dependence beyond the Kolmogorov wavenumber, i.e. a spectral slope of -1 . Measurements in the laboratory (e.g. Gibson \& Schwarz 1963) and in the ocean (e.g. Grant et al. 1968) were subsequently reported to be in accord with this prediction. On the other hand, measurements by Gargett (1985) in the ocean were found not to exhibit a $k^{-1}$ spectral range (see, however, discussion by Gibson 1987, 1991). The same result was noted in passive scalar mixing measurements in shear layers (Komori et al. 1989) and turbulent jets (Miller \& Dimotakis 1991b; Miller 1991). Despite adequate resolution in those experiments, no $k^{-1}$ range was found at high spatial wavenumbers, or, to be exact, temporal frequencies. In addition, questions have been raised about the universality, if not validity, of the $k^{-1}$ spectrum predictions at high wavenumbers, in high Schmidt number turbulent fluid flows (Dimotakis \& Miller 1990).

The issue of spatial vs. temporal spectra should be recognized here. The classical theories cited deal with spatial spectra. One could argue, therefore, that comparisons of measurements of temporal spectra with predictions of spatial spectra cannot be made directly. Two points are noted in response. First, the overwhelming majority of experimentally obtained spectra reported to be in accord with the theoretical predictions have, in fact, been temporal. Second, developing flows, such as a turbulent jet, are not (statistically) spatially homogeneous over the range of spectral scales of interest. The notion of a spatial spectrum and the assumption of a statistically spatially homogeneous turbulent field, for such flows, are questionable. Temporal spectra, derived from point measurements, do not have to contend with this issue.

\section{Experiment}

The experiments investigated the scalar (concentration) field of round, axisymmetric, momentum-dominated, turbulent jets issuing from contoured nozzles into a large, quiescent discharge tank. The measurements were performed in the far field, on the centreline of the jet. Details of the experimental apparatus have appeared previously (Miller \& Dimotakis 1991a,b; Miller 1991), so only a brief overview will be presented here.

The experimental facility consists of three major parts: the jet plenum, nozzle, and delivery system; a large reservoir that acts as the discharge tank; and the diagnostics, consisting of an argon-ion laser, focusing optics, collection optics, detector, signalprocessing electronics, and the subsequent data processing. The working fluid is water, and the scalar is a laser dye (disodium fluorescein) which is homogeneously premixed

† The outer flow Reynolds number values were kindly provided by S. Saddoughi (private communication). 
with the jet plenum fluid. The resulting Schmidt number,

$$
S c \equiv \frac{v}{\mathscr{D}}
$$

where $v$ is the kinematic viscosity (of water) and $\mathscr{D} \simeq 5.2 \times 10^{-6} \mathrm{~cm}^{2} \mathrm{~s}^{-1}$ the estimated aqueous species diffusivity of the fluorescein dye, is $1.9 \times 10^{3}$ (Ware et al. 1983, p. 280).

The jet flow was established and maintained by pressurizing a downward oriented jet plenum with gas. Both sonically metered and blow-down, nearly-constant-pressure, gas delivery configurations were used. The internal exit diameter of the jet nozzle is $2.5 \mathrm{~mm}$ $(0.1 \mathrm{in}$.). The rectangular discharge tank is square in cross-section, approximately $2 \mathrm{~m}$ high and $1 \mathrm{~m}$ across its base. The tank bottom is over 600 nozzle diameters downstream. Large glass windows on all four sides provided optical access (see Miller 1991 for further details).

The illumination source was an argon-ion laser (Coherent Innova 90). The particular unit was custom selected for its low AM noise figure $(\sim-95 \mathrm{~dB})$ over the frequency range of interest in these experiments. It was operated at a power of $3.5 \mathrm{~W}$ in the light-regulation mode. The beam was spatially filtered, expanded, collimated, and subsequently focused to a small waist located on the centreline of the jet. A low dye concentration was used in the jet plenum $\left(\sim 10^{-6} \mathrm{M}\right)$, with correspondingly substantially lower concentrations at the measuring stations. A more detailed discussion of this and related issues may be found in Miller \& Dimotakis (1991b) and Miller (1991). The emitted fluorescence intensity was found to be proportional to the local scalar (dye) concentration, $c(x, t)$, averaged over the extent of the measurement volume.

The fluorescence emitted from the measurement volume was collected through a narrow slit spatial filter. The beam profile and the slit width defined a small, spatially averaging volume, roughly spherical in shape and of extent (diameter) $\ell_{a} \approx 50 \mu \mathrm{m}$, as estimated by Gaussian beam optics and verified by direct observation using a cathetometer. Our spectral data also yielded an independent estimate of this quantity, as will be discussed below.

A photomultiplier tube (RCA 8645) was used to detect the fluorescence emitted from this volume. Its output signal was amplified by a low-noise transimpedance amplifier (custom-designed by Dr D. Lang), low-pass filtered using a third-order Butterworth filter, digitized, sampled with some margin with respect to the Nyquist frequency, and stored for subsequent processing.

The measurements to be discussed here were made in the far field, on the axis of the jet, for jet Reynolds numbers in the range of

$$
1.25 \times 10^{4} \leqslant R e \equiv \frac{u_{j} d}{v} \leqslant 7.2 \times 10^{4},
$$

where $u_{j}$ is the jet nozzle exit velocity, $d=2.54 \mathrm{~mm}$ is the nozzle exit diameter, and $v$ is the kinematic viscosity. The water temperature in these experiments was $(20 \pm 2)^{\circ} \mathrm{C}$. The jet nozzle exit velocity was estimated in terms of the volume-discharge velocity, by measuring the time required to discharge a fixed volume from the jet plenum (cf. Miller 1991, tables A.2 and A.4, for actual values). The Reynolds number values were estimated to be known within 5-10\%. Data were also recorded at both lower and higher Reynolds numbers. The lower Reynolds number jets, however, behaved substantially differently, by any of a number of criteria, and were not accepted as representing bona fide turbulence (Miller \& Dimotakis 1991b). In the other limit, the 


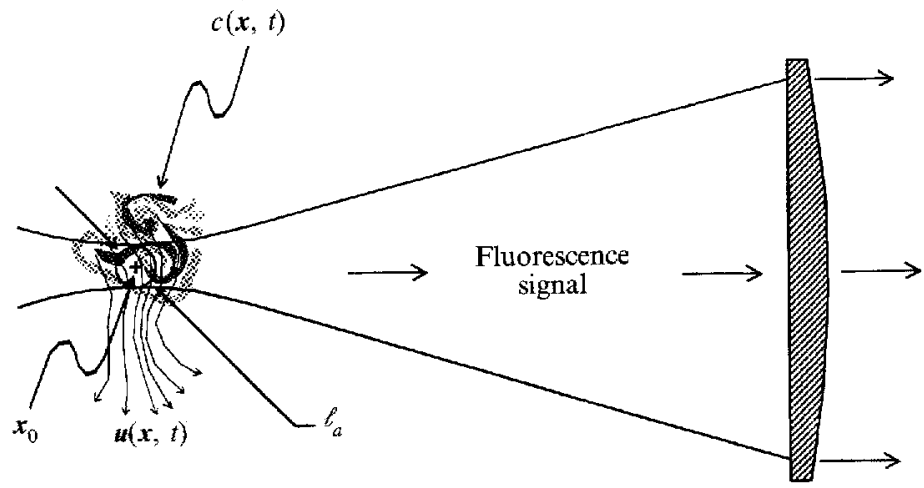

FIGURE 1. Sketch of jet-fluid concentration field $c(x, t)$, convected through the measurement volume of extent $\ell_{a}$.

jet at the higher Reynolds number $\left(R e=10.2 \times 10^{4}\right)$ produced a distinct hissing sound. This was probably generated by the transient dilatation and subsequent oscillations of small air bubbles caused by the rapid reduction in pressure in such bubbles as they exited the nozzle, or by cavitation in the jet near-field region, or both (note that the plenum gauge pressure is quadratic with Reynolds number). See discussion and references in Blake (1986, pp. 452-453) and Young (1989, pp. 205-207), for example. As a result, the $R e=10.2 \times 10^{4}$ jet was exposed to different near-field conditions and will not be included in the discussion below. See Miller (1991) for further documentation of the data.

Finally, constraints dictated by resolution and statistical convergence, vis-à-vis total number of large-scale structures captured and length-of-run considerations, led to measurement stations in the range,

$$
100 \leqslant \frac{x}{d} \leqslant 305,
$$

where $x$ is the distance from the nozzle exit.

\section{Scalar power spectrum estimation}

The fluorescence signal, $\phi(t)$, representing the photon flux incident on the photodetector, is a linear function of the spatial integral over the measurement volume of the convected local jet-fluid concentration field, $c(x, t)$. It produces a signal that can be approximated by a convolution over $c(t)=c\left(x_{0}, t\right)$, the jet-fluid concentration at, say, the centre of the measurement volume, $x_{0}$, i.e.

$$
\phi(t) \simeq \int_{-\infty}^{\infty} h_{a}\left(t-t^{\prime}\right) c\left(t^{\prime}\right) \mathrm{d} t^{\prime} \equiv h_{a}(t) \otimes c(t) .
$$

In this expression, $h_{a}(t)$ models the impulse response of the measurement process, i.e. the temporal signal that would be measured if a spatial delta function of dye was convected through the measurement volume at the local flow velocity. See figure 1.

The fluorescence output $\phi(t)$, along with fluctuations contributed by the small laser intensity fluctuations, convected residual non-uniformities in the jet plenum dye concentration, photon shot noise, electronic noise generated by the signal-processing chain, etc., was processed by the Butterworth low-pass filter to produce the total 


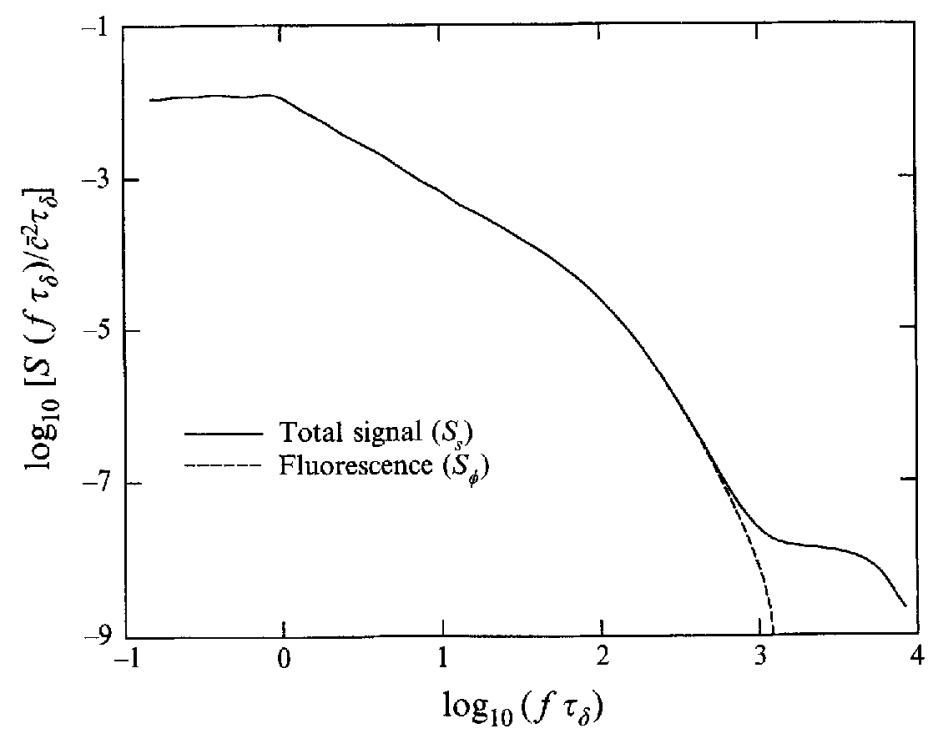

Figure 2. Sample spectrum of the total signal (solid line: fluorescence + noise) and estimated fluorescence spectrum (dashed line: fluorescence), derived from measurements at $x / d=100$, $R e=1.25 \times 10^{4}$. Frequency scaled by $\tau_{\delta}(x)$, the local large-scale passage time.

signal

$$
s(t)=h_{L P}(t) \otimes \phi(t)+n(t)=h(t) \otimes c(t)+n(t),
$$

that was digitized and stored. In this expression, $h(t) \equiv h_{L P}(t) \otimes h_{a}(t)$ is the total system transfer function and $n(t)$ is the total, low-pass-filtered noise.

Assuming that the system noise $n(t)$ can be modelled as uncorrelated with the local dye concentration time history $c(t)$, the spectrum $S_{s}(\omega)$ of $s(t)$ can be expressed in terms of the spectrum $S_{\phi}(\omega)$ of $\phi(t)$ and the spectrum $S_{n}(\omega)$ of the (low-pass filtered) noise $n(t)$, i.e.

$$
S_{s}(\omega) \simeq S_{\phi}(\omega)+S_{n}(\omega)
$$

where, from (4),

$$
S_{\phi}(\omega) \simeq\left|H_{a}(\omega)\right|^{2} S_{c}(\omega),
$$

with $H_{a}(\omega) \equiv \mathscr{F} \mathscr{T}\left\{h_{a}(t)\right\}$, the Fourier transform of $h_{a}(t)$. This allows us to relate the total signal spectrum, $S_{s}(\omega)$, to the desired scalar fluctuation spectrum, $S_{c}(\omega)$, of $c(t)$, i.e.

$$
S_{s}(\omega) \simeq|H(\omega)|^{2} S_{c}(\omega)+S_{n}(\omega) \simeq\left|H_{a}(\omega)\right|^{2} S_{c}(\omega)+S_{n}(\omega),
$$

where $H(\omega) \equiv \mathscr{F} \mathscr{T}\{h(t)\}=H_{L P}(\omega) H_{a}(\omega)$.

For these experiments, the knee of the Butterworth low-pass filter was set substantially higher than the range of frequencies contained in $S_{\phi}(\omega)$. Its main purpose was to band-limit the noise and de-alias the digitized measurements, allowing the noise floor to be determined, as will be illustrated in the spectra presented below. This is the reason why the modulus squared of $H_{L P}(\omega)$, the transfer function of the low-pass filter, can be ignored in (7) and (8), and wherever it multiplies $S_{\phi}(\omega)$ and $S_{c}(\omega)$.

Figure 2 illustrates these relations by comparing the spectrum $S_{s}(\omega)$ of the total signal $s(t)$, i.e. fluorescence signal $\phi(t)$ plus noise $n(t)$, with $S_{\phi}(\omega)$, the spectrum of the fluorescence signal alone. The latter was calculated by subtracting the estimated noise 
spectrum, $S_{n}(\omega)$, from the total spectrum $S_{s}(\omega)$. Recalling (6), we have

$$
S_{\phi}(\omega) \simeq S_{s}(\omega)-S_{n}(\omega) \text {. }
$$

The noise spectrum was assumed to be white, as was found to be the case in separate measurements of this quantity (see also Dowling, Lang \& Dimotakis 1989, for examples). Nevertheless, one can appreciate that the result is not sensitive to the assumed shape of the noise spectrum at low frequencies, where $S_{\phi}(\omega)$ dominates. The data processed to produce the spectra in figure 2 were recorded at $x / d=100$, for $R e=1.25 \times 10^{4}$. Note the high dynamic range of the total signal spectrum, i.e. the (logarithmic) difference of the low-frequency power to noise-floor power. Note also that the span to one-half the (scaled) sampling frequency is well beyond the noise-cross-over frequency. As can be seen, the noise floor is well determined.

The spectra in figure 2, and throughout this paper, are normalized by $\bar{c}^{2}$, the square of the local mean value of $c(t)$, multiplied by the local large-scale passage time, $\tau_{\delta}(x)$, and plotted in terms of the circular frequency, $f$, scaled by $\tau_{\delta}(x)$. In these coordinates, their integral produces the normalized variance,

$$
\frac{c^{\prime 2}}{\bar{c}^{2}}=\int_{0}^{\infty}\left[\frac{S_{c}\left(f \tau_{\delta}\right)}{\bar{c}^{2} \tau_{\delta}}\right] \mathrm{d} f \tau_{\delta}
$$

The local large-scale passage time, $\tau_{\delta}$, is given by

$$
\tau_{\delta}(x) \equiv \frac{\delta(x)}{u_{c l}(x)},
$$

where

$$
\delta(x) \simeq 0.41 x
$$

is the local outer scale of the flow, here identified with the (measured) mean transverse extent, i.e. visual width, of the conical region enveloping the jet fluid (Miller 1991, Appendix D), and $u_{c l}(x)$ is the mean centreline velocity. The latter was estimated from the relation

$$
\frac{u_{c l}(x)}{u_{j}}=6.2 \frac{d}{x-x_{j}},
$$

where $u_{j}$ is the jet exit velocity and $x_{j}$ the jet (virtual) origin, as recommended by Chen \& Rodi (1980). The virtual origin, $x_{j}$, for these experiments was, in all cases, less than two nozzle diameters and, in view of the distances of the measuring stations from the jet nozzle, was neglected. This spectrum and frequency scaling was found to produce similarity with respect to the downstream coordinate, $x / d$, in the analysis of scalar spectra measured in gas-phase jets (Dowling \& Dimotakis 1990).

The spectrum $S_{c}(\omega)$ of the scalar fluctuations $c(t)$ can, at least formally, be estimated by solving (7), i.e.

$$
S_{c}(\omega) \simeq \frac{S_{\phi}(\omega)}{\left|H_{a}(\omega)\right|^{2}}
$$

Combining with (6), we obtain a result in terms of experimentally estimated quantities,

$$
S_{c}(\omega) \simeq \frac{S_{s}(\omega)-S_{n}(\omega)}{\left|H_{a}(\omega)\right|^{2}} .
$$

As long as the fluorescence signal, $\phi(t)$, and the local jet-fluid concentration, $c(t)$, are linearly related, an equation of the form of (7) represents the most general expression for this relation. However, while (7) follows from (4), the converse is not true. Equation 
(4) is more restrictive, also prescribing a definite phase relation between $c(t)$ and $\phi(t)$. Fortunately, these (unknown) phase relations do not enter in the corresponding spectra. We may conclude that (7) and (12) provide good approximations for the corresponding spectra, even though it may not be possible to determine a (fixed) time-domain kernel, $h_{a}(t)$ in (4), that provides a correct description of the time history of the fluorescence signal, $\phi(t)$.

The estimation of the scalar spectrum $S_{c}(\omega)$, in the frequency range influenced by the averaging performed by the measurement process, requires knowledge of the $H_{a}(\omega)$ transfer function. This can be estimated, in turn, by noting that it is dominated by a pole corresponding to the transit time of the flow through the measurement volume, i.e.

with

$$
H_{a}(\omega) \approx \frac{1}{1+\mathrm{i} \omega \tau_{a}}
$$

$$
\tau_{a} \approx \frac{\ell_{a}}{u_{c l}(x)} \text {. }
$$

As a consequence, fluorescence spectra, $S_{\phi}(\omega)$, and local concentration fluctuation spectra, $S_{c}(\omega)$, can be expected to depart from each other at normalized (circular) frequencies (cf. (10)) in the neighbourhood of

$$
f \tau_{\delta}(x) \approx \frac{\delta(x)}{2 \pi \ell_{a}}
$$

and above, i.e. at a scaled frequency that is independent of the Reynolds number.

Performing two experiments, at different spatial resolutions, under as identical flow conditions as feasible, it is possible to compare the two spectra, e.g. $p=1,2$,

$$
S_{s_{p}}(\omega) \simeq\left|H_{p}(\omega)\right|^{2} S_{c}(\omega)+S_{n_{p}}(\omega)
$$

corresponding to two different locations of the dominant pole, at, say, $\tau_{a}=\tau_{1}$ and $\tau_{a}=\tau_{2}>\tau_{1}$, i.e. for $p=1,2$,

$$
H_{p}(\omega) \approx \frac{1}{1+\mathrm{i} \omega \tau_{p}}
$$

If the scalar spectrum, $S_{c}(\omega)$, can be assumed to be identical in the two experiments and the corresponding noise floors are determined separately in each case, the ratio of the two estimated fluorescence spectra,

$$
\frac{S_{\phi_{1}}(\omega)}{S_{\phi_{2}}(\omega)} \simeq \frac{S_{s_{1}}(\omega)-S_{n_{1}}(\omega)}{S_{s_{2}}(\omega)-S_{n_{2}}(\omega)} \equiv G\left(\omega ; \tau_{1}, \tau_{2}\right)
$$

will be given by

$$
G\left(\omega ; \tau_{1}, \tau_{2}\right) \approx \frac{1+\left(\omega \tau_{2}\right)^{2}}{1+\left(\omega \tau_{1}\right)^{2}}=\left\{\begin{array}{cl}
1 & \text { for } \omega \ll 1 / \tau_{2}, \\
\left(\tau_{2} / \tau_{1}\right)^{2} & \text { for } \omega \gg 1 / \tau_{1},
\end{array}\right.
$$

independently of the, as yet unknown, scalar spectrum, $S_{c}(\omega)$.

From one such pair of experiments, at $x / d=100$ and $R e=1.25 \times 10^{4}$, two such fluorescence spectra, $S_{\phi_{1}}(\omega)=S_{s_{1}}(\omega)-S_{n_{1}}(\omega)$ and $S_{\phi_{2}}(\omega)=S_{s_{2}}(\omega)-S_{n_{2}}(\omega)$, were obtained. They are plotted in figure 3 (dotted lines). The fluorescence spectrum with the larger high-frequency content is the one plotted in figure 2.

The ratios $G\left(\omega ; \tau_{1}, \tau_{2}\right)$ for the pair of spectra, measured at $x / d=100$, at $\operatorname{Re}=$ $1.25 \times 10^{4}$ (circles) and a pair at $\operatorname{Re}=2.55 \times 10^{4}$ (squares), respectively, are plotted in 


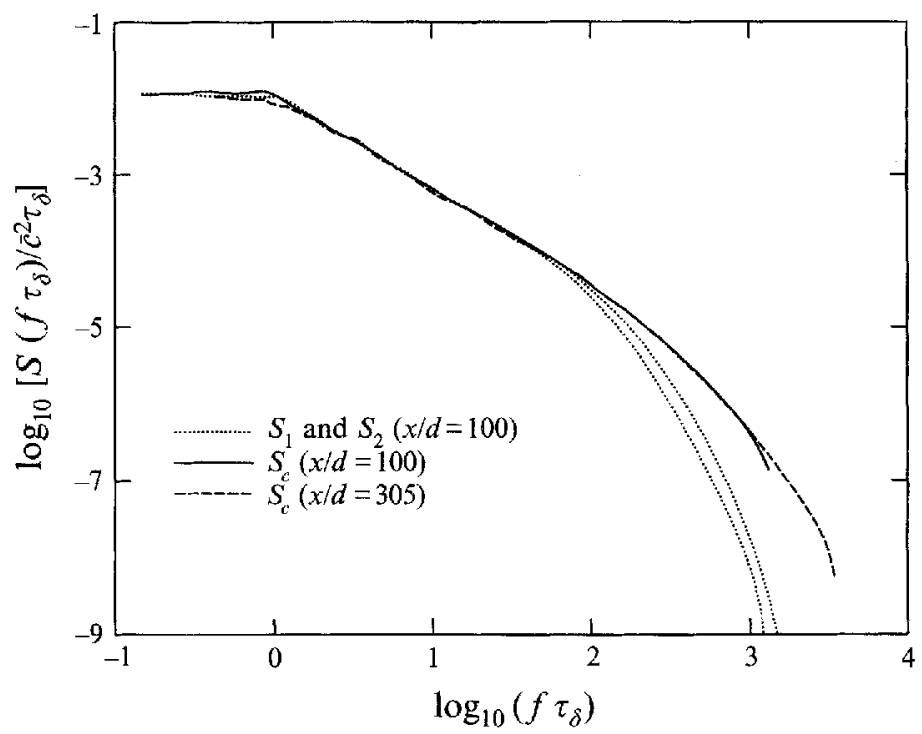

FIGURE 3. Dotted lines: fluorescence spectra estimated from measurements at $x / d=100$ and $R e=1.25 \times 10^{4}$ at two spatial resolutions. Solid line: estimated concentration spectrum (equation (12)) at $x / d=100$ and $R e=1.25 \times 10^{4}$. Dashed line: estimated concentration spectrum at $x / d=305$ and $\operatorname{Re}=1.2 \times 10^{4}$.

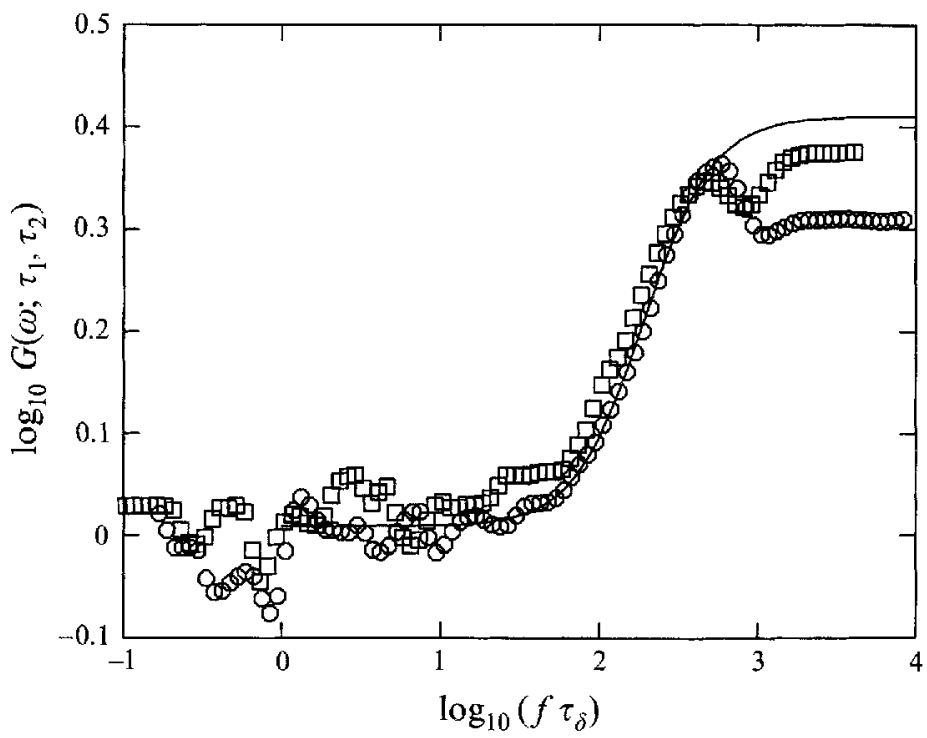

FIGURE 4. Computed ratio, $G\left(\omega ; \tau_{1}, \tau_{2}\right)$, of fluorescence spectra at $x / d=100$ (equation (16)). Circles: $\operatorname{Re}=1.25 \times 10^{4}$, squares: $\operatorname{Re}=2.55 \times 10^{4}$, solid line: least-squares fit for $\tau_{1}$ and $\tau_{2}$.

figure 4. As can be seen, the ratios of spectra measured at different Reynolds numbers are very nearly the same, in accord with the analysis outlined above, even though, as we will see below, the spectra themselves are Reynolds-number-dependent and quite different.

The curve (solid line) in figure 4 is a least-squares fit for $\tau_{1}$ and $\tau_{2}$ to the lower Reynolds number data that were characterized by the higher signal-to-noise ratio, in the frequency range $1.0 \leqslant f \tau_{\delta} \leqslant 2.8$. The lower limit of the fit range is chosen so as to exclude (the small) run-to-run variations at frequencies well below those affected 


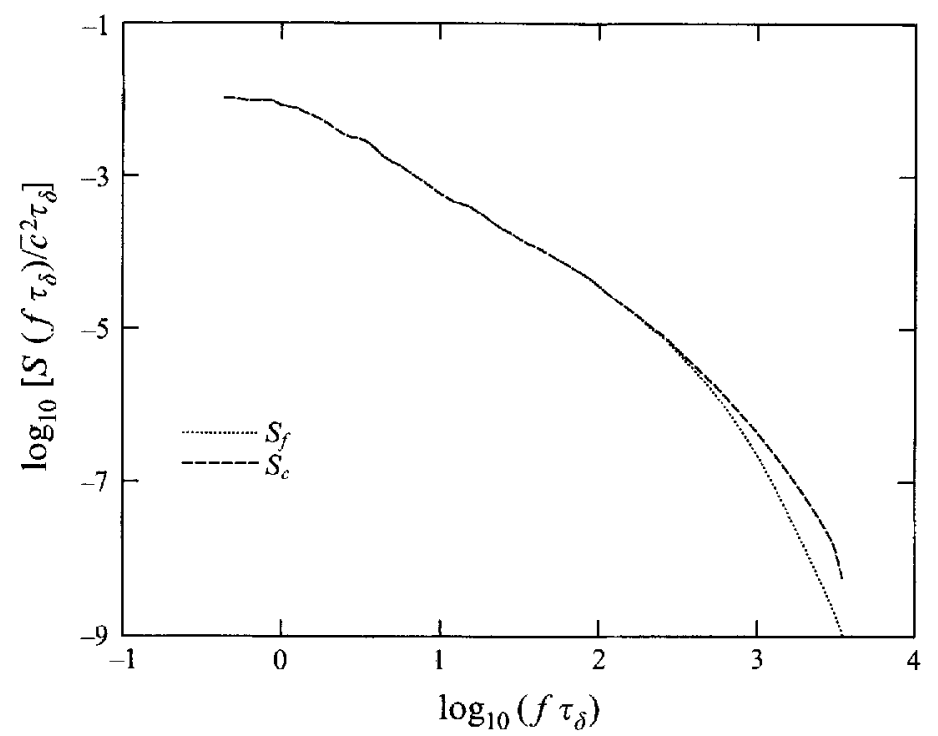

FIGURE 5. Dotted line: fluorescence spectrum. Dashed line: estimated concentration spectrum. Data recorded at $x / d=305$ and $R e=1.2 \times 10^{4}$.

by the spatial averaging. The upper frequency limit is dictated by the less than unity signal-to-noise ratio at higher frequencies yet (cf. figure 2). The values for $\tau_{1}$ and $\tau_{2}$ estimated by this procedure were

$$
2 \pi \tau_{1} / \tau_{\delta} \simeq 4.2 \times 10^{-3} \quad \text { and } \quad 2 \pi \tau_{2} / \tau_{\delta} \simeq 6.6 \times 10^{-3},
$$

respectively. This corresponds to an effective spatial extent of the measurement volume of $\ell_{a} \simeq 69 \mu \mathrm{m}$ for the smaller of the two (equation (14)), in reasonable accord with the previously cited, visually estimated value of $\sim 50 \mu \mathrm{m}$ using the cathetometer. This value was used to calculate the concentration spectrum, $S_{c}(\omega)$, at $x / d=100$ and $R e=1.25 \times 10^{4}$, plotted as the solid line in figure 3. It was computed from the fluorescence spectrum recorded at the higher resolution, using (12) with the estimated single-pole transfer function $H_{a}(\omega)$ of (13), at $\tau_{a}=\tau_{1}$.

The effective pole locations for data recorded at $x / d=305$ were more difficult to estimate. At $x / d=305$, the higher relative spatial resolution pushed the poles closer to the noise cross-over point. On the other hand, at $x / d=305$, the (logarithmic) difference between the fluorescence and estimated concentration spectra was much smaller over the frequency range of interest. Figure 5 plots the fluorescence spectrum (dotted line) at $x / d=305$ and $R e=1.2 \times 10^{4}$ as well as the estimated concentration spectrum (dashed line). As can be seen, the effects of compensation, in this case, are much smaller (cf. the difference at, say, $\log _{10}\left(f \tau_{\delta}\right) \simeq 2.7$ in figures 3 and 5 ). The estimated concentration spectrum at $x / d=305$ in figure 5 is the one plotted as a dashed line in figure 3.

The jet-fluid concentration spectra to be discussed below were all estimated in this fashion. The power spectra themselves, in this work, were computed numerically using a power spectral density estimation methodology that has evolved over the past ten years, or so. A documentation of some of its earlier features can be found in Dowling (1988). Briefly, the power spectral density estimation program computes spectra of data files by means of FFT methods, and incorporates Hanning windowing, contiguous record overlapping, and parabolic detrending, among other features. Records up 
to $2^{17}$ points can be accommodated. For spectra known to be smooth, the program can provide third-octave ( $\simeq 1 / 10$ decade) Gaussian filtering, sampled at 20 points per decade, to produce the final spectra. This feature was used for all the spectra plotted in this paper, not so much for smoothing, but to reduce the number of points to a manageable level for plotting purposes $\left(2^{16}=65536\right)$.

The values of $\tau_{\alpha}$, the transfer function time constant used in the compensation calculations, were fixed for all the data measured at each $x / d$ axial location (equation (17), for measurements at $x / d=100$, depending on which slit width was used to record the fluorescence data). A fixed pair of values was also used for all the data measured at $x / d=305$.

In summary, the effects of compensation were limited to $\log _{10}\left(f \tau_{\delta}\right) \gtrsim 2.0$ for data recorded (in the high-resolution configuration) at $x / d=100$, and to $\log _{10}\left(f \tau_{\delta}\right) \gtrsim$ 2.5 for data similarly recorded at $x / d=305$, as dictated by spatial resolution considerations (temporal resolution was not an issue for any of the measurements). Conversely, the spectra can be regarded as resolved, with respect to the finite spatial and temporal resolution of these measurements, without the benefits of compensation, for scaled frequencies below these (Reynolds-number-independent) values (cf. (14)).

In contrast, the spectral extent, as limited by finite signal-to-noise ratio considerations, i.e. up to frequencies such that the signal spectrum $S_{s}(\omega)$ is sufficiently higher than the noise spectrum $S_{n}(\omega)$ (cf. (6)), is a function of the flow velocity. As a consequence, as will be evident from the data to be presented below, the highest scaled frequency for which the concentration spectra could be determined was a function of the flow Reynolds number and $x / d$.

\section{Results and discussion}

The agreement between the concentration spectra at $x / d=100$ (solid line) and $x / d=305$ (dashed line) in figure 3, up to frequencies limited by signal-to-noise ratio considerations, should be noted. A similar independence of the scaled spectra with downstream location was also found to hold in gas-phase jets (Dowling \& Dimotakis 1990), where the relatively larger diffusion scales, for $S c \approx 1$ and comparable Reynolds numbers, made it possible to estimate the concentration spectra with enough spatial resolution directly, obviating the need for the compensation scheme employed here.

It is useful to plot the product of the concentration spectrum with $f^{5 / 3}$, as is commonly done. A spectrum described by a $-5 / 3$ power law yields a horizontal line over the $-5 / 3$ frequency range when plotted in this fashion. The product of the concentration spectrum and $\left(f \tau_{\delta}\right)^{5 / 3}$, derived from the data recorded at $x / d=100$ for $R e=1.25 \times 10^{4}$, and $x / d=305$ for $R e=1.2 \times 10^{4}$, is plotted in figure 6 as the solid and dashed curves, respectively. Also plotted, for reference, is a straight line with a slope (logarithmic derivative) of $2 / 3$, corresponding to the high Schmidt number, $k^{-1}$, theoretically predicted spectrum by Batchelor (1959).

To connect the measurement scales with the flow microscales, we may use the definition of the Kolmogorov (1941) scale, i.e.

$$
\lambda_{K} \equiv\left(\frac{v^{3}}{\varepsilon}\right)^{1 / 4},
$$

where $\varepsilon$ is the kinetic energy dissipation rate. This can be estimated from the on-axis 


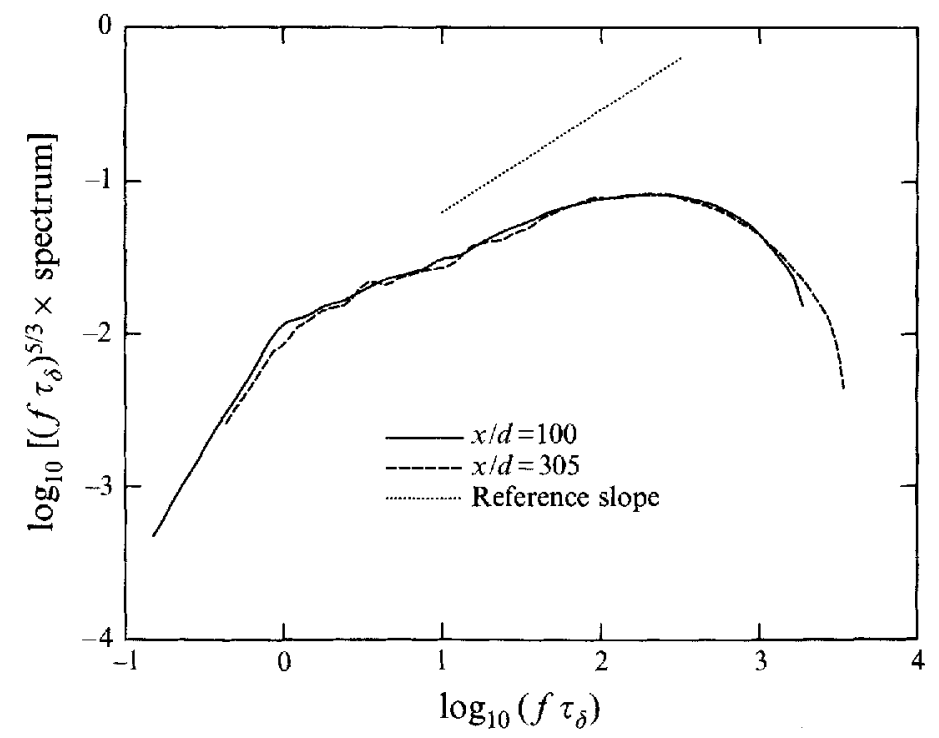

FIGURE 6. Frequency-scaled concentration spectra. Solid line: $x / d=100$ and $R e=1.25 \times 10^{4}$. Dashed line: $x / d=305$ and $R e=1.2 \times 10^{4}$. Dotted line: reference line at a $2 / 3$ slope, corresponding to a $k^{-1}$ spectrum.

relation proposed by Friehe, Van Atta \& Gibson (1971) for a jet,

$$
\varepsilon \simeq 48\left(\frac{u_{j}}{d}\right)^{3}\left(\frac{d}{x-x_{j}}\right)^{4}
$$

to obtain

$$
\frac{\lambda_{K}}{\delta} \approx 0.95 \operatorname{Re}^{-3 / 4}
$$

At $x / d=305$ and $R e=1.2 \times 10^{4}$, for example, $\lambda_{K} \simeq 257 \mu \mathrm{m}$, as compared to the spatially averaging length of the measurements, of $\ell_{a} \simeq 50-70 \mu \mathrm{m}$.

To convert the Kolmogorov spatial scale to the frequency scaling employed in the spectra in the present discussion, we note that,

$$
\begin{aligned}
k \lambda_{K}=\omega \tau_{K} & =2 \pi f \tau_{K} \\
& =2 \pi\left(\frac{\tau_{K}}{\tau_{\delta}}\right) f \tau_{\delta} \\
& =2 \pi\left(\frac{\lambda_{K}}{\delta}\right) f \tau_{\delta} .
\end{aligned}
$$

The Kolmogorov (circular) frequency, corresponding to the wavenumber where $k \lambda_{K}=1$, is then given by

$$
2 \pi\left(\frac{\lambda_{K}}{\delta}\right) f_{K} \tau_{\delta}=1
$$

or

$$
\log _{10}\left(f_{K} \tau_{\delta}\right)=\log _{10}\left(\frac{\delta}{2 \pi \lambda_{K}}\right) \simeq \frac{3}{4} \log _{10}(R e)-0.78
$$

where (20) was employed to obtain the last relation. The Batchelor (1959) scalar 


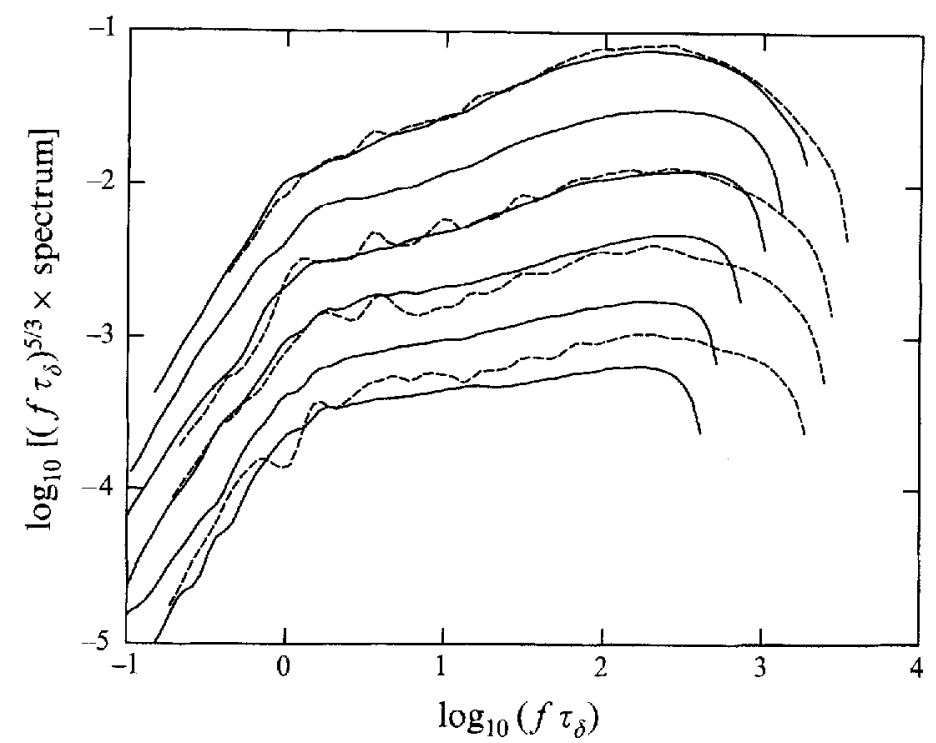

FIGURE 7. Frequency-scaled concentration spectra. Solid lines: $x / d=100$ data $\left(1.25 \leqslant \operatorname{Re} \times 10^{-4} \leqslant 7.2\right)$. Dashed lines: $x / d=305$ data $\left(1.2 \leqslant \operatorname{Re} \times 10^{-4} \leqslant 6.5\right)$. Individual spectra are offset by $-2 \log _{10}\left(R e / R e_{0}\right)$, with $R e_{0}=1.2 \times 10^{4}$, for clarity.

diffusion scale, $\lambda_{B}$, can then be estimated as

$$
\hat{\lambda}_{B} \approx \lambda_{K} / S c^{1 / 2} \simeq \lambda_{K} / 43 \text {, }
$$

corresponding to the Schmidt number value for the dye used in these experiments. By way of example, for $R e=1.2 \times 10^{4}$ this leads to an estimate for the scaled frequencies of

$$
\log _{10}\left(f_{K} \tau_{\delta}\right) \simeq 2.3 \text { and } \log _{10}\left(f_{B} \tau_{\delta}\right) \simeq 3.9
$$

where $f_{B} / f_{K}=\lambda_{K} / \lambda_{B}$. Comparing with the (collapsed) spectra depicted in figure 6 , we see that the spectra are resolved in the present experiments to frequencies extending about a decade beyond the Kolmogorov-scale passage frequency, $f_{K}$, at least at this Reynolds number.

We note, however, that the spatial scales where the influence of viscosity is felt can be taken as, roughly, an order of magnitude larger than the Kolmogorov scale (cf. Chapman 1979). At a given convection velocity, this yields a passage frequency of the largest viscous scales of $f_{v} \approx 0.1 f_{K}$. One can similarly argue that scalar diffusivity will also manifest itself at spatial scales roughly an order of magnitude larger than the Batchelor scale, corresponding to a passage frequency of $f_{\mathscr{Q}} \approx 0.1 f_{B}$. This then leads to estimates for the viscous and scalar diffusion passage frequencies, for $R e=1.2 \times 10^{4}$, given by

$$
\log _{10}\left(f_{v} \tau_{\delta}\right) \approx 1.3 \quad \text { and } \quad \log _{10}\left(f_{\mathscr{Z}} \tau_{\delta}\right) \approx 2.9
$$

The solid lines in figure 7 plot spectra derived from measurements at $x / d=100$, for $R e \times 10^{-4}=1.25,1.76,2.55,3.6,5.1$, and 7.2. The decrease in the maximum scaled frequency in the $x / d=100$ spectra (as limited by signal to noise), with increasing Reynolds number, is evident. The dashed lines plot spectra measured at $x / d=305$, for $\operatorname{Re} \times 10^{-4}=1.2,2.4,4.0$, and 6.5 . The highest frequency captured in the $x / d=305$ spectra is a much weaker function of Reynolds number, at this station. 


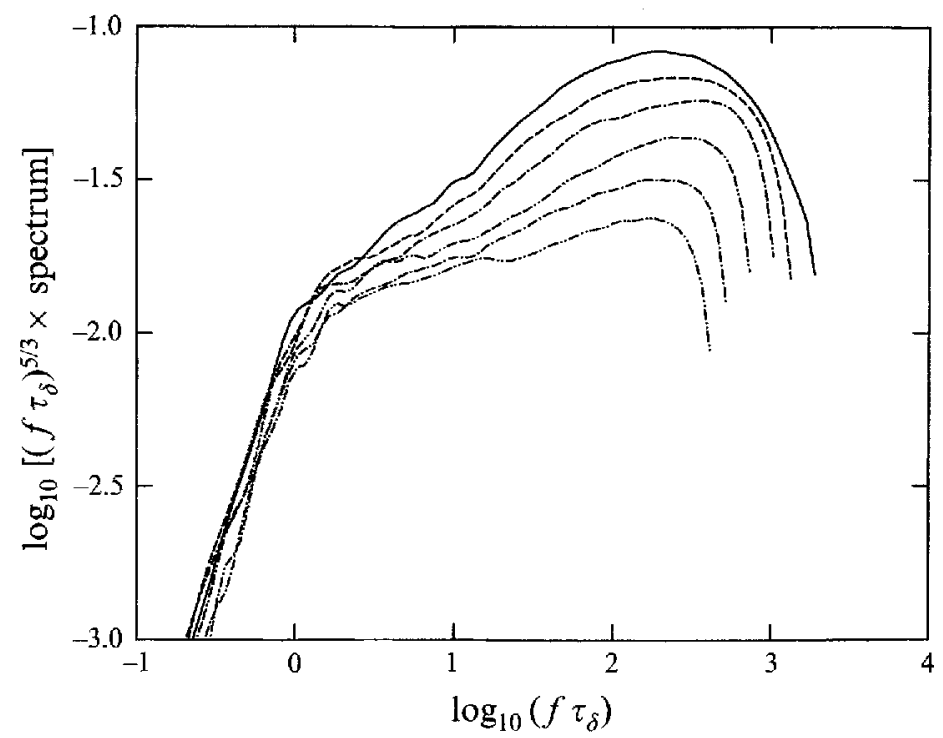

FIGURE 8. Frequency-scaled concentration spectra derived from data at $x / d=100$ and $1.25 \leqslant \operatorname{Re} \times 10^{-4} \leqslant 7.2$ (no offsets). See text for line styles.

Individual spectra for both $x / d$ stations are plotted offset by $-2 \log _{10}\left(R e / R e_{0}\right)$, with $R e_{0}=1.2 \times 10^{4}$, to aid in visualizing the evolution of trends with Reynolds number.

The statistical convergence of the $x / d=100$ spectra is better than the $x / d=305$ spectra, owing to the much larger number of large-scale structures captured at the upstream station. On the other hand, the higher relative spatial resolution at $x / d=305$ allowed the spectrum to be estimated to a higher (scaled) frequency. With this in mind, the agreement between the (scaled) spectra at $x / d=100$ and $x / d=305$ is very good for all the cases for which data were recorded at the same, or nearly the same, Reynolds number at the two stations.

Following the transition out of the large-scale frequency regime $\left(f \tau_{\delta} \approx 1\right)$, the spectra appear to be described by a power law with an exponent that is increasing from roughly 1.2 to 1.5 in absolute value, with increasing Reynolds number. This progression with Reynolds number is easier to discern in figure 8 , which plots the concentration spectra at $x / d=100$, for $R e \times 10^{-4}=1.25$ (solid line), 1.76 (dashed line), 2.55 (dot-dash-dash), 3.6 (dot-dot-dash-dash), 5.1 (dot-dash), and 7.2 (dot-dotdash), with no offsets. The extent of the power-law regime can be seen to increase slightly with increasing Reynolds number.

Estimates of the spectrum slope in the power-law region vs. Reynolds number, derived from the spectra in figure 7 , as well as three estimates derived from data at $x / d=170$, are plotted in figure 9 (cf. Miller 1991, figure 5.2, and related discussion). A similarly increasing spectrum slope (in absolute value) with Reynolds number was also noted in measurements of gas-phase spectra (Dowling \& Dimotakis 1990), for $\operatorname{Re} \times 10^{-4}=0.5,1.6$, and 4.0 .

As can be seen by sighting along the spectra in figure 8 , the power-law region is followed by a different regime at higher frequencies yet. This regime does not support the Batchelor (1959) $k^{-1}$ prediction that should apply for over a decade and a half in frequency in this case (recall $S c \approx 1.9 \times 10^{3}$ for these experiments). This can be seen in figure 6 , which includes a dotted line with a reference slope of $2 / 3$, corresponding to a $k^{-1}$ spectrum. The spectra at increasing Reynolds number depart even further 


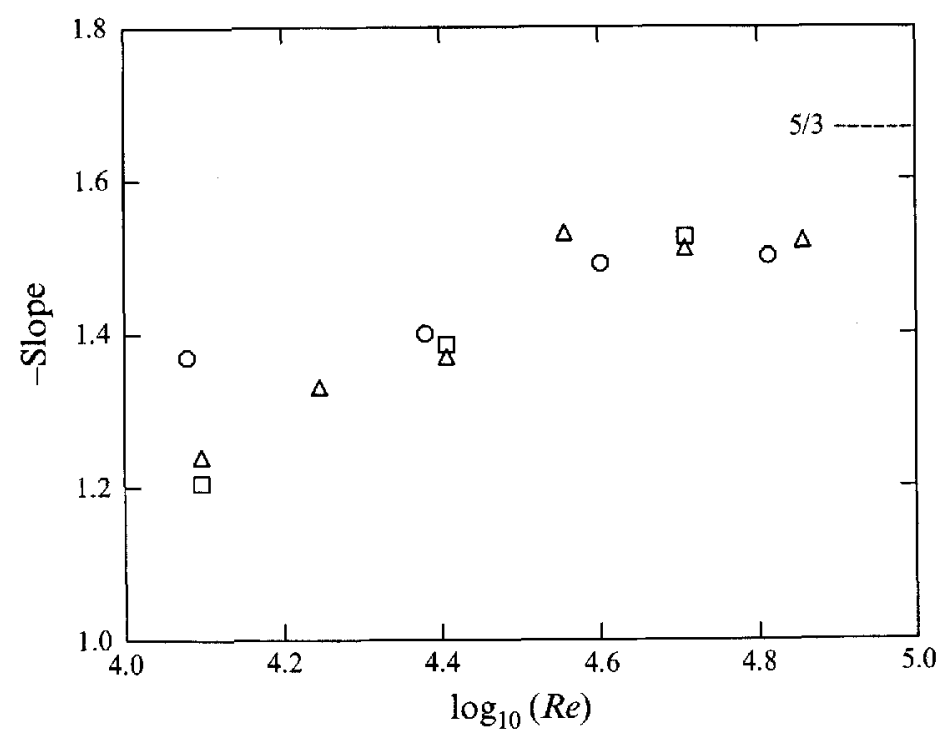

FIGURE 9. Average spectrum slopes $v$. Re, from data at $x / d=100$ (triangles), $x / d=170$ (squares), and $x / d=305$ (circles). Dashed line at top right corresponds to a $-5 / 3$ spectrum slope.

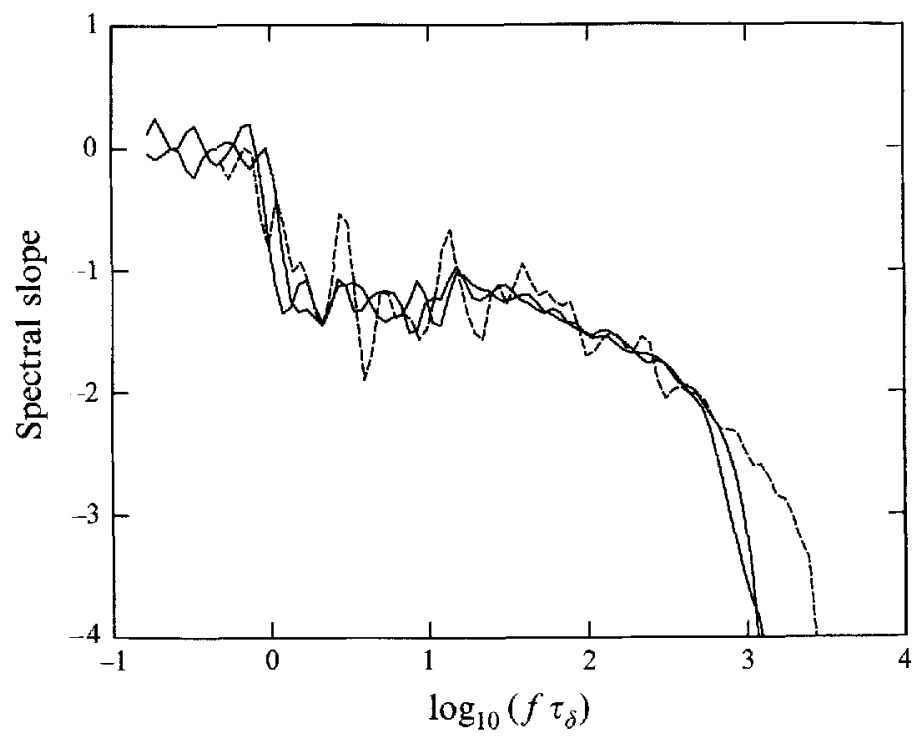

FIGURE 10. Local spectrum slope (logarithmic derivative). Solid lines: data at $x / d=100$ and $R e=1.25 \times 10^{4}$. Dashed line: $x / d=305, R e=1.2 \times 10^{4}$.

from this slope, as can be seen by comparing the $R e=1.2 \times 10^{4}$ spectrum with those at higher Reynolds numbers in figure 8. It is important to note that this conclusion also extends to frequencies below $\log _{10}\left(f \tau_{\delta}\right) \simeq 2$, i.e. frequencies that are unaffected by spatial/temporal resolution, compensation, and signal-to-noise considerations.

To facilitate the study of this higher-frequency regime, the slopes (logarithmic derivatives) of the spectra were also computed. A plot of spectrum slopes, for the lower Reynolds number data, appears in figure 10. These were derived from the two $x / d=100, R e=1.25 \times 10^{4}$ spectra in figure 3 (solid lines) and the $x / d=305$, $R e=1.2 \times 10^{4}$ spectrum (dashed line). Their comparison helps assess issues of 


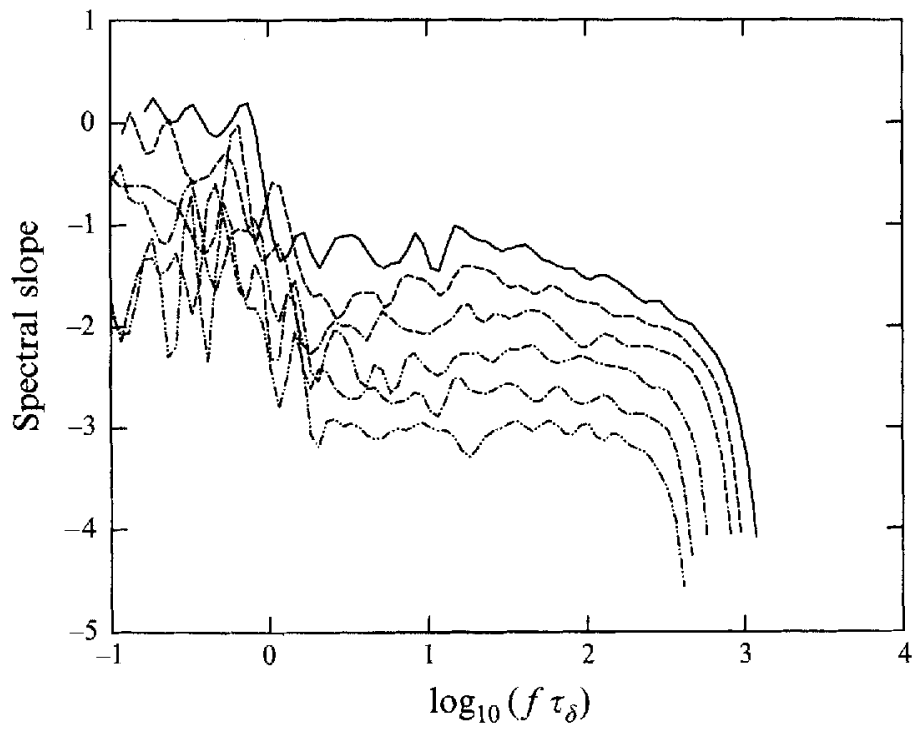

FIGURE 11. Local slope (logarithmic derivative) of spectra from data at $x / d=100$ and $1.25 \leqslant R e \times 10^{-4} \leqslant 7.2$. Spectra offset as in figure 7 . Line types as in figure 8 .

statistical convergence and confidence in this more delicate statistic, as well as the effects of spatial resolution and the applied compensation.

The plots in figure 10 suggest a frequency-dependence of the slope of the spectra that is close to a straight line, in these coordinates. The straight line appears to be a good representation for frequencies below $\log _{10}\left(f \tau_{\delta}\right) \approx 2$, for which the effects of compensation were negligible, even for the $x / d=100$ data (cf. figure 3). For frequencies above $\log _{10}\left(f \tau_{\delta}\right) \simeq 2$, the same straight line also describes the behaviour for both the $x / d=100$ and $x / d=305$ data, which were affected by resolution (and compensation) to a different extent (cf. figures 3 and 5).

A straight line for the slope (logarithmic derivative) of the spectrum corresponds to a spectrum that is parabolic in log-log coordinates, or log-normal in linear coordinates, i.e.

$$
S_{c}\left(f \tau_{\delta}\right) \propto \exp \left\{-\frac{1}{2}\left[a \ln \left(f \tau_{\delta}\right)+b\right]^{2}\right\} .
$$

This expression, rather than a $k^{-1}$ power law, seems to provide a better description of our jet-fluid concentration spectra at high frequencies, over a range of frequencies at least as large as $S c^{1 / 2}$, i.e. a decade and a half, in this case. Notably, this spectral regime can be seen to span a range of frequencies, $1.2 \lesssim \log _{10}\left(f \tau_{\delta}\right) \lesssim 2.8$, in reasonable accord with the passage frequencies for the viscous and scalar diffusion scales, at this Reynolds number (cf. (24b)).

Figure 11 plots the local slope (logarithmic derivative) of the spectra in figure 8 . The offset scheme employed in figure 7 and line types employed in figure 8 were also used here. Straight lines can be seen to be a good representation for the spectrum slope at high frequencies, with Reynolds-number-dependent values of the parameters $a$ and $b$ in (25). The end of the power-law regime and the beginning of the log-normal range can be seen to shift to higher frequencies with increasing Reynolds number. Our data admit a Kolmogorov scaling for the Reynolds number dependence of this transition frequency, i.e. $\sim R e^{3 / 4}$, but corresponding to the convection (passage frequency) of a physical scale roughly 80 times larger than the estimated Kolmogorov scale (cf. 
(18) and discussion following), i.e. frequencies that are close to the estimated viscousscale transition frequency, $f_{v}$ (cf. $(24 b)$ ). This transition is not very well defined, however, and other Reynolds number scaling possibilities cannot be ruled out. It is not possible to assess the Reynolds number dependence of the upper bound of this frequency regime, owing to resolution and signal-to-noise limitations with increasing Reynolds number.

Several other reports in the literature document temperature, or scalar species, concentration spectra in turbulent flows with 'bump' features at high wavenumbers similar to the ones found in the experiments reported here. These include: measurements of temperature fluctuations in turbulent jets (Clay 1973; Chevray \& Tutu 1978), of smoke concentration in turbulent jets (Shaughnessy \& Morton 1977), temperature measurements in tidal channels (Grant et al. 1968; Gargett 1985), concentration measurements in grid turbulence (Gibson \& Schwarz 1963), and temperature measurements in an atmospheric boundary layer (Williams \& Paulson 1978). In addition, similar bump features have been observed in both velocity- and temperature-derivative spectra (Champagne et al. 1977; Williams \& Paulson 1978), as well as in velocity spectra in large, well-resolved boundary layers (Mestayer 1982; Saddoughi \& Veeravalli 1994).

Those experiments did not report scalar spectra with any significant $k^{-1}$ region at high wavenumbers. Two explanations have been, typically, offered to date: that probe resolution was insufficient, or that the Schmidt/Prandtl number was not large enough. The resolution of our measurements (recall comparison of compensated and uncompensated spectra measured at different $x / d$ stations, figures 3 and 6 ), is sufficient to have resolved at least the beginning, if not a good part, of a $k^{-1}$ region, if one existed. Therefore, probe resolution is not a factor in these measurements and does not impact on this result. Secondly, the Schmidt number in our experiments was high, specifically, of order 2000 . In contrast, bumps qualitatively similar to the ones found in our spectra were documented in spectra computed from flows with Prandt numbers as low as 0.7 (temperature in air). According to the Batchelor (1959) theory, the extent of the viscous-convective $k^{-1}$ range should scale like $S c^{1 / 2}$, i.e. a factor of 53 more than the Prandtl number 0.7 case. Nevertheless, no $k^{-1}$ region was found.

Finally, we should note that we are aware of at least one report in the literature of an extensive -1 slope region in a scalar spectrum, by Nye \& Brodkey (1967). A fibre-optic probe was used in those experiments to measure the concentration of dye injected into turbulent pipe flow, and found 1.5 decades of -1 slope in the concentration spectrum, 36 diameters downstream, but no $-5 / 3$ slope (or inertial) range. It is possible, however, that this lower-wavenumber behaviour arises for similarity reasons in outer boundary-layer and pipe-flow spectra, where one can argue that $\omega y / U$, where $\omega$ is the temporal frequency, $y$ is the distance from the wall, and $U$ is the outer flow velocity, is the appropriate dimensionless frequency in the dynamics. Perry \& Abell (1975) put such a scaling argument forth to explain a large $\omega^{-1}$ region in their own turbulent-pipe-flow longitudinal-velocity spectra.

\section{Conclusions}

This work has investigated temporal scalar (jet fluid concentration) power spectra on the centreline of high Schmidt number turbulent jets, in the Reynolds number range $1.2 \leqslant R e \times 10^{-4} \leqslant 7.2$. Our spectra exhibit a power-law regime at frequencies above the local large-scale passage frequency, with a Reynolds-number-dependent exponent increasing (in absolute value) from roughly -1.2 to -1.5 over the Reynolds 
number range investigated. This corroborates a similar finding for gas-phase jetfluid concentration spectra measured at comparable Reynolds numbers (Dowling \& Dimotakis 1990). At higher frequencies, the spectra are well represented by a lognormal relation with Reynolds-number-dependent coefficients. While our data admit a Kolmogorov-like scaling for the beginning of the log-normal region in the spectrum, i.e. $\sim R e^{3 / 4}$, other possibilities cannot be ruled out.

Our results are at odds with the classical picture of high Schmidt number scalar spectra. In particular, our spectral data do not exhibit a $-5 / 3$ power-law regime, even though recorded at Reynolds numbers where such behaviour has previously been reported for high Schmidt number jet-fluid scalar spectra (e.g. Becker, Hottel \& Williams 1967). Nevertheless, the data do not preclude a $-5 / 3$ spectral slope in the limit of higher Reynolds numbers yet (cf. figure 9). Finally, despite adequate resolution and signal-to-noise ratio, our data do not support the Batchelor (1959) $k^{-1}$ power-law prediction for the spectrum describing small-scale dynamics. Specifically, we found no constant $k^{-1}$ slope at high frequencies, or even a spectral slope that locally attains a value of -1 .

On the whole and as noted above, however, our scalar spectra are similar, in many respects, to previous scalar as well as to velocity spectra derived by other experimenters in a variety of flows. In conjunction with those data and analyses, the current results raise further questions about the universal descriptions of scalar spectra, and their applicability to some of the canonical flows, including turbulent jets.

We would like to acknowledge contributions by Dan Lang and David Dowling to the experiments. This work was supported by AFOSR Grants Nos. 83-0213, 88-0155, and F49620-92-J-0290, as well as GRI Contract No. 5087-260-1467.

\section{REFERENCES}

BATCHELOR, G. K. 1959 Small-scale variation of convected quantities like temperature in turbulent fluid. Part 1. General discussion and the case of small conductivity. $J$. Fluid Mech. 5, 113-133.

BATChelor, G. K., Howells, I. D. \& TownSENd, A. A. 1959 Small-scale variation of convected quantities like temperature in turbulent fluid. Part 2. The case of large conductivity. J. Fluid Mech. 5, 134-139.

Becker, H. A., Hottel, H. C. \& Williams, G. C. 1967 The nozzle-fluid concentration field of the round turbulent, free jet. $J$. Fluid Mech. 30, 285-303.

BLAKE, W. K. 1986 Mechanics of Flow-Induced Vibration, Vol. II. Academic.

Champagne, F. H., Friehe, C. A., La Rue, J. C. \& Wyngaard, J. C. 1977 Flux measurements, flux estimation techniques and fine scale turbulent measurements in the surface layer over land. $J$. Atmos. Sci. 34, 515-530.

Chapman, D. R. 1979 Computational aerodynamics development ond outlook. AIAA J. 17, 12931313.

Chin, C. J. \& Rodi, W. 1980 Vertical Turbulent Buoyant Jets. A Review of Experimental Data. Pergamon.

Chevray, R. \& Tutu, N. K. 1978 Conditional measurements in a heated turbulent jet. In Structure and Mechanics of Turbulence II (ed. H. Fiedler). Lecture Notes in Physics, vol. 76, pp. 73-84. Springer.

ClaY, J. P. 1973 Turbulent mixing of temperature in air, water, and mercury. $\mathrm{PhD}$ thesis, U. C. San Diego.

CoRrsin, S. 1951 On the spectrum of isotropic temperature fluctuations in isotropic turbulence. $J$. Appl. Phys. 22, 469-473.

Dimotakis, P. E. \& Miller, P. L. 1990 Some consequences of the boundedness of scalar fluctuations. Phys. Fluids A 2, 1919-1920. 
Dowling, D. R. 1988 Mixing in gas phase turbulent jets. $\mathrm{PhD}$ thesis, California Institute of Technology.

Dowling, D. R. \& Dimotakis, P. E. 1990 Similarity of the concentration field of gas-phase turbulent jets. J. Fluid Mech. 218, 109-141.

Dowling, D. R., Lang, D. B. \& Dimotakis, P. E. 1989 An improved laser-Rayleigh scattering photodetection system. Exps. Fluids 7, 435-440.

Friehe, C. A., Van AtTA, C. W. \& Gibson, C. H. 1971 Jet turbulence: Dissipation rate measurements and correlations. AGARD Turbulent Shear Flows CP-93, 18.1-7.

GargetT, A. E. 1985 Evolution of scalar spectra with the decay of turbulence in a stratified fluid. J. Fluid Mech. 159, 379-407.

GiBsoN, C. H. 1987 Fossil turbulence and intermittency in sampling oceanic mixing processes. $J$. Geophys. Res. 92, C5, 5383-5404.

GiBson, C. H. 1991 Kolmogorov similarity hypotheses for scalar fields: sampling intermittent turbulent mixing in ocean and galaxy. Proc. R. Soc. Lond. A 434, 149-164.

Gibson, C. H. \& SCHWARz, W. H. 1963 The universal equilibrium spectra of turbulent velocity and scalar fields. J. Fluid Mech. 16, 365-384.

Grant, H. L., Hughes, B. A., Vogel, W. M. \& Mollliet, A. 1968 The spectrum of temperature fluctuations in turbulent flow. J. Fluid Mech. 34, 424-442.

Kolmogorov, A. N. 1941 Local structure of turbulence in an incompressible viscous fluid at very high Reynolds numbers. Dokl. Akad. Nauk SSSR 30, 299.

Komori, S., Kanzaki, T., Murakami, Y. \& Ueda, H. 1989 Simultaneous measurements of instantaneous concentrations of two species being mixed in a turbulent flow by using a combined laser-induced fluorescence and laser-scattering technique. Phys Fluids A 1, 349-352.

MESTAYER, P. 1982 Local isotropy and unisotropy in a high-Reynolds-number turbulent boundary layer. J. Fluid Mech. 125, 457-503.

MiLler, P. L. 1991 Mixing in high Schmidt number turbulent jets. $\mathrm{PhD}$ thesis, California Institute of Technology.

Miller, P. L. \& Dimotakis, P. E. $1991 a$ Stochastic geometric properties of scalar interfaces in turbulent jets. Phys Fluids A 3, 168-177.

Miller, P. L. \& Dimotakis, P. E. $1991 b$ Reynolds number dependence of scalar fluctuations in a high Schmidt number turbulent jet. Phys. Fluids A 3, 1156-1163.

Monin, A. S. \& YaGlom, A. M. 1975 Statistical Fluid Mechanics: Mechanics of Turbulence, vol. 2 (ed. J. Lumley). MIT Press.

NYE, J. O. \& BRoDKEY, R. S. 1967 The scalar spectrum in the viscous-convective subrange. J. Fluid Mech. 29, 151-163

Oвоuкov, А. М. 1962 Some specific features of atmospheric turbulence. J. Fluid Mech. 13, 77-81.

Perry, A. E. \& Abrell, C. J. 1975 Scaling laws for pipe-flow turbulence. J. Fluid Mech. 67, 257-271.

Saddoughi, S. G. \& VeEravalLi, S. V. 1994 Local isotropy in turbulent boundary layers at high Reynolds number. J. Fluid Mech. 268, 333-372.

Shaughnessy, E. J. \& MoRTon, J. B. 1977 Laser light-scattering measurements of particle concentration in a turbulent jet. $J$. Fluid Mech. 80, 129-148.

WARE, B. R., CYR, D., GorTI, S. \& LANNI, F. 1983 Electrophoretic and frictional properties of particles in complex media measured by laser light scattering and fluorescence photobleaching recovery. Measurement of Suspended Particles by Quasi-Elastic Light Scattering, pp. 255-289. Wiley.

Williams, R. N. \& Paulson, C. A. 1978 Microscale temperature and velocity spectra in the atmospheric boundary layer. J. Fluid Mech. 83, 547-567.

YouNG, F. R. 1989 Cavitation. McGraw-Hill. 